\title{
Research on the Fluctuation Characteristics of China's Urban Economy Under the Control of Novel Coronavirus Pneumonia (COVID-19)
}

\author{
Aili $\mathrm{Du}^{1}$ and Lei Wang ${ }^{2, *}$ \\ ${ }^{1}$ School of Accounting, Shandong Technology and Business University, China \\ ${ }^{2}$ School of Mathematics and Information Science, Shandong Technology and Business University, China \\ *Corresponding author. Email: wl@sdtbu.edu.cn
}

\begin{abstract}
The novel coronavirus pneumonia characterized by affecting widely and spreading quickly brings a significant impact on the economy in China. In order to avoid a substantial economic downturn, we should pay attention to its shock on economic activities as well as controlling the pandemic effectively. Based on the big data at city level, we use a combination model composed of difference-in-difference method and event study method to measure and evaluate the impact of the novel coronavirus pneumonia on urban economic activity quantitatively and scientifically. The main conclusions are as follows. The pandemic of the novel coronavirus pneumonia significantly restrains economic activities in cities, causing that the overall intensity of economic activity in cities across the country during the two weeks after Spring Festival in 2020 are decreased by $48.4 \%$ compared to the same period in 2019, and the negative effects of the pandemic on economic activity are more prominent in cities with large population migration before the Spring Festival and high dependence on migrant workers.
\end{abstract}

Keywords: Novel coronavirus pneumonia (COVID-19), Pandemic control, Urban economy, Population mobility.

\section{INTRODUCTION}

Novel coronavirus pneumonia (COVID-19) cases which occurred in Wuhan, Hubei province at the end of 2019 spread to the whole country quickly. By February 23, 2020, 77150 confirmed cases had been reported and 635531 close contacts had been traced in 31 provinces and Xinjiang production and Construction Corps. The pandemic which broke out during the Chinese Lunar New Year with large scale and high intensity of population mobility, leading to the rapid spread of the pandemic, has a very negative impact on the economic and social development.

Compared with the other public health emergencies including the SARS in 2003, COVID-19 affect a wider population and spread faster, causing a significant impact on the Chinese economy, especially the retail, restaurant and tourism industries. While effectively controlling the pandemic situation, we should also pay close attention to its impact on China's economic activities and avoid a sharp fall in the economy.
Adhering to the "two lines" - prevention and control of pandemic and economic construction- at the same time, in accordance with the principle of prevention and control at different levels, paying close attention to the changes of the pandemic situation, and focusing on the economic construction work are of great significance to alleviate the negative impact of the pandemic on the economy. Therefore, a reasonable assessment of the impact of COVID-19 on economic activities using big data at the level of subdivided cities and cutting-edge econometric methods can provide an important practical basis for the government to formulate targeted policies and measures for pandemic prevention and control and economic recovery.

\section{REVIEW OF THE LITERATURE}

Domestic and foreign scholars have conducted an indepth study on the impact of public health emergencies such as pandemic diseases or infectious diseases on economic and social development. For example, Philipson [1] believes that the spread of infectious 
diseases will increase the cost of disease prevention of residents, which is not conducive to the accumulation of personal wealth. In order to reduce the burden of residents' medical expenditure, Gersovitz et al. [2] point out that government intervention is the key factor to break through the personal prevention dilemma. Zhu Ling [3] believes that a scientific and perfect social medical security system is helpful to protect the health safety of residents and the economic security of the country. In terms of comparative research and interdisciplinary analysis, Lei Juan [4] points out that the prevention and control of the pandemic should respect the rights of patients and prudently use judicial tools through the analysis of the compulsory medical system for infectious diseases in the United States. Krieger [5] and Song Leiming [6] put forward the idea of combining Anthropology with epidemiology from an interdisciplinary perspective to promote the smooth implementation of public health practice. Liu Dehai et al. [7] propose that the government should be cautious and flexible in formulating prevention and control policies in response to major public health emergencies such as SARS.

An important reference for COVID-19 research is SARS in 2003. The research on SARS related issues can be basically divided into four aspects: First, from the perspective of public health mechanism, it reflects on the imbalance of resource allocation and functional dislocation of public health management departments in China[8,9,10]; The second is to focus on the impact of the spread of SARS on the economy, including economic growth, employment and inflation ${ }^{[11,12,13]}$. For example, according to the estimation of Cai Fang and Wang Meiyan[12], the SARS pandemic reduces the total employment by 1.27 million to 5.3 million in 2003 .

Based on the above literature, most of the attention on COVID-19 has been focused on estimating the speed of virus transmission and predicting the number of infected people, while less literature analyzes the impact of COVID-19 on economic growth. In the studies of the impact of infectious or pandemic diseases on China's economy, due to the limitations of data or methods, most of them are qualitative descriptions and lack of empirical support. Based on the real-time and high frequency data of the intensity of travel and population migration at various city levels in China, the impact of COVID-19 on urban economic activities in China is quantitatively measured in this paper. We expect to mine valuable information from the flow of the urban population and economic activities to reflect the actual situation of the impact of the pandemic on China's macro-economy specifically.

\section{SOURCE OF DATA}

\subsection{Source of Data and Calculation of Variables}

The data in this paper are mainly from Baidu migration big data which includes various kinds of population migration indexes. Taking the urban administrative divisions as the boundary, we calculate the daily scale index of population moving out, daily scale index of population moving in and urban travel intensity index of 388 cities (including municipalities directly under the central government, prefecture level cities, regions, States, alliances, autonomous counties, etc.) during the Spring Festival transportation in 2019 and 2020, and then the scale index and intensity index of each city can be compared horizontally. Since COVID-19 burst around the Spring Festival, abundant data can be obtained from cities during the Spring Festival travel rush in 2020 and the same period of the lunar calendar in 2019 for studying the impact of COVID-19 on economic activity in cities and across the country. For addition, data on confirmed cases of COVID-19 comes from Baidu's real-time big data report on COVID-19 and the website of the Chinese Center for Disease Control and Prevention.

The main relevant data information we can get from the big data of Baidu migration is about "chunyun"- Spring Festival transportation-which refers to the large-scale, nationwide traffic peak phenomenon around the Chinese Lunar New Year. Generally speaking, "chunyun" lasts for 40 days, 15 days before and 25 days after the Spring Festival. For example, the 2019“chunyun" last from January 21 to March 1,2019, and the 2020 Spring Festival travel rush last from January 10 to February 18,2020.

As mentioned above, the impact of COVID-19 on economic activity is first reflected on population. In order to fully reflect its impact, we calculate a number of variables for each city for analysis in this paper. The calculation methods of the main variables are listed in Table 1. 
Table 1. Calculation of city variables based on Baidu migration big data

\begin{tabular}{|c|c|c|c|}
\hline Name & Symbol & Calculation methods & Sample time point \\
\hline Employment gap & Gap & $\begin{array}{c}\text { Ratio of the scale index of moving out } 15 \text { days before } \\
\text { the Spring Festival to the scale index of moving in } \\
\text { before the Spring Festival }\end{array}$ & 2019,2020 \\
\hline Rate of work resumption & Rework & $\begin{array}{l}\text { Ratio of the mean of the travel intensity in the city in } \\
\text { the second week after the Spring Festival to the mean } \\
\text { of the travel intensity in the city in the second week } \\
\text { before the Spring Festival }\end{array}$ & 2019,2020 \\
\hline $\begin{array}{c}\text { Scale of population moving } \\
\text { out before the Spring } \\
\text { Festival }\end{array}$ & Moveout & $\begin{array}{l}\text { Sum up the scale of population moving out every day } \\
\text { from the beginning of the chunyun to the Spring } \\
\text { Festival ( } 15 \text { days before the Spring Festival) }\end{array}$ & 2019,2020 \\
\hline $\begin{array}{c}\text { Scale of population moving } \\
\text { in before the Spring } \\
\text { Festival }\end{array}$ & Movein & $\begin{array}{l}\text { Sum up the scale of population moving in every day } \\
\text { from the beginning of the chunyun to the Spring } \\
\text { Festival ( } 15 \text { days before the Spring Festival) }\end{array}$ & 2019,2020 \\
\hline $\begin{array}{l}\text { Dependence on migrant } \\
\text { workers }\end{array}$ & Demworker & $\begin{array}{c}\text { Ratio of the scale of population moving out before the } \\
\text { Spring Festival to the scale of population moving in } \\
\text { before the Spring Festival }\end{array}$ & 2019,2020 \\
\hline Economic activity index & Activity & $\begin{array}{l}\text { Ratio of the travel intensity in the city every day during } \\
\text { the chunyun to the average of the three maximum } \\
\text { values of travel intensity in the city one month before } \\
\text { the Spring Festival }\end{array}$ & $\begin{array}{l}\text { Two weeks before and } \\
\text { after the Spring } \\
\text { Festival in } 2019 \text { and } \\
2020\end{array}$ \\
\hline
\end{tabular}

\subsection{Descriptive Statistical Analysis}

\subsubsection{Feature Statistics about COVID-19}

In this paper, the characteristics of the COVID-19 pandemic are described based on the data of confirmed COVID-19 cases in all cities (excluding Hong Kong, Macao and Taiwan) as of February 14, 2020 (form retention for request). It should be noted that in order to avoid the influence of extreme values, Wuhan is not included in the statistics. The number of the cities with more than 1000 confirmed cases is 6, compared with $1.64 \%$ of all cities, but the number of confirmed cases as high as 10689 , accounts for $38.37 \%$ of the total number of confirmed cases. The result fully reflects the high agglomeration phenomenon of COVID-19 in some cities, especially in Hubei province. In contrast, the number of the cities with fewer than 10 confirmed cases is 149 and the total number of confirmed cases in the group is 526 , accounting for $1.89 \%$ of the total number of confirmed cases.

\subsubsection{Employment Gaps in Cities}

After the spread of COVID-19 to the whole country, restrictions on population movement, traffic restrictions and other measures are adopted to prevent and control the pandemic. The employment gaps in most cities in 2019 are small and around zero, basically realizing the balance between population moving in and moving out before and after the Spring Festival. In addition, in 2019,334 of the 388 cities have negative employment gaps, that is, the population moving in after the Spring Festival is slightly larger than the population moving out before the Spring Festival. One possible reason is that more rural labor force flows into cities to seek job opportunities after the Spring Festival, which meets the demand for labor force in cities. However, in 2020, due to the outbreak of the pandemic, the population moving out before the Spring Festival could not return normally, resulting in employment gaps in all cities. The employment gaps in almost all cities are positive, with a maximum of 196.91 and an average of 12.85 .

\subsubsection{Rates of Work Resumption in Cities}

In the context of COVID - 19, the employment gap has a close relationship with the rate of work resumption. Based on the calculated the rate of work resumption and the number of confirmed cases in each city, the scatter plot is drawn to reflect the relationship between them. So as to avoid the extreme value, cities with confirmed cases less than 1000 as of February 14, 2020 are selected for analysis, as shown in Figure 1. 


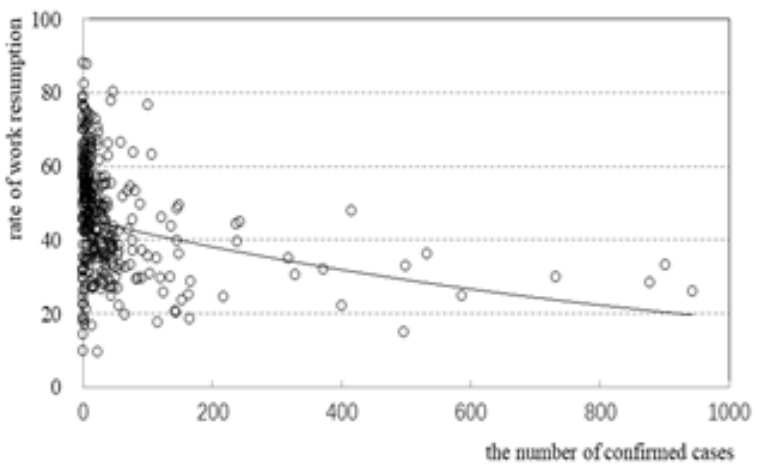

Figure 1 the number of confirmed cases in each city and the rate of work resumption

As can be seen from Figure 1, there is basically a negative relationship between confirmed COVID-19 cases and the rate of work resumption. In other words, cities with more confirmed cases have a lower rate of work resumption. In cities with a big number of confirmed cases, the government tends to adopt stricter traffic control measures and delay the resumption of work and production, in order to take the control of the pandemic as the top priority, which is in line with the reality. Under the influence of the pandemic and the government's control, the rates of work resumption of all cities are low, with an average return rate of $46.36 \%$ and 213 cities with a rate of work resumption of less than 50\% after the Spring Festival in 2020. It should also be noted that among cities with similar number of confirmed COVID-19 cases, there are also significant differences in the rates of work resumption. Among the 62 cities with 1 to 5 confirmed cases as of February 14, 2020 , the maximum and minimum rates of work resumption are $82.43 \%$ and $17.02 \%$, respectively, and the standard deviation is $14.47 \%$.

\section{EMPIRICAL ANALYSIS}

\subsection{Construction of a Difference-in-difference Model}

COVID-19 can be regarded as a natural experiment, and research on the impact of the pandemic needs to find the experimental group and the control group. Since COVID-19 occurred around the Spring Festival in 2020, each city becomes an experimental group affected by the pandemic; While the economic activity of each city around the 2019 Spring Festival was not affected by the pandemic, the data of each city in 2019 provides a good control group for itself. The difference-in-difference method (DID), is an effective method to deal with natural experimental problems and has become an appropriate tool for assessing the effects of the pandemic.

Based on the economic activity index data of each city during the two weeks before and after the Spring
Festival in 2019 and 2020, we construct a standard difference-in-difference method (DID) model to explore the impact of COVID-19 on economic Activity, in the form of:

Activity $_{\text {it }}=\alpha_{1}+\alpha_{2} D g+\alpha_{3} D t+\alpha_{4} D g \cdot D t+u_{i t}$

Where $i$ and $t$ are respectively the symbols of city and time; Dg is a dummy variable used to distinguish the experimental group from the control group. Dt is a time dummy variable, used to divide the time of the event; uit is a random disturbance term; $\alpha 1 \sim \alpha 4$ are parameters to be estimated. In model (1), the setting methods of the two dummy variables are shown in Formula (2).

$D g=\left\{\begin{array}{lr}0, & \text { control group } \\ 1, & \text { experimental group }\end{array}\right.$,

$D t=\left\{\begin{array}{lc}0, & \text { before the pandemic } \\ 1, & \text { after the pandemic }\end{array}\right.$

The objective of applying the difference-in-difference method (DID) is to compare the differences between the experimental group and the control group before and after the outbreak of the pandemic. $\alpha 4$ - the interaction term coefficient between the grouped dummy variable and the time dummy variable -is the key to reflect the "net effect" of the pandemic event.

\subsection{Empirical Results Analysis}

In this paper, we calculate average economic activity index each 10 working days before and after the Spring Festival in 2019 and 2020 respectively and plot in figure 5. The economic activity of each city remains relatively stable before and after the Spring Festival in 2019, while under the influence of COVID-19, the economic activity of each city in 2020 shows a significant change before and after the Spring Festival, and the economic activity index drops significantly after the Spring Festival.

To further test the impact of the pandemic on urban economic activities, the difference-in-difference (DID) model (1) is estimated in this paper and the estimation results are:

Activity $_{i t}=0.912+0.004 \mathrm{Dg}-0.005 \mathrm{Dt}-$ $0.484 D g \cdot D t+\hat{u}_{i t}$

$\mathrm{t}=(539.60)(1.72)(-1.95)(-143.20)$

s.e. $=(0.002)(0.002)(0.003)(0.003)$

$\mathrm{N}=15520 \quad \mathrm{R}^{2}=0.799$

The estimation results of the difference-in-difference model (DID) show that the interaction term coefficient between the grouped dummy variable and the time dummy variable is significantly -0.484 , indicating that 
COVID-19 has significantly suppressed economic activity in cities. Since the economic activity index is referenced to 1 , the results suggest that the pandemic reduced the intensity of economic activity in cities nationwide by $48.4 \%$ in the two weeks after the Spring Festival in 2020 compared to the two weeks of working days after the Spring Festival in 2019. Because COVID19 pandemic is a contagious disease, controlling the source of infection and cutting off the transmission route are effective measures to prevent the spread of the pandemic. After the outbreak, local governments takes strong measures such as "city closure" and "quarantine" to restrict the large-scale movement of people. In this context, the dates of workers returning to work and enterprises' resumption of work are all delayed, which not only restricts the consumption and investment activities on the demand side, but also restricts the production behavior of enterprises on the supply side, and thus has a negative impact on the economic activities in the city.

Besides the travel restrictions imposed by the government, the pandemic has triggered negative emotions such as public concern and anxiety, leading people to subjectively refuse to go out and spend the holidays at home, which has become a special phenomenon during the Chinese Lunar New Year in 2020. This reflects the increased awareness of infectious diseases and the effectiveness of government policies to prevent and control the pandemic. However, according to time series factor decomposition and seasonal adjustment studies, the Spring Festival period is usually a golden period for domestic retail and tourism consumption surges. In other words, there is a Spring Festival factor in the time series, and the resulting "the Spring Festival effect" is a driving force for economic growth. After the Spring Festival in 2020, the phenomenon of "home holiday" which affects the normal operation of the economy, especially the service sector with high share in the economy, makes the "Spring Festival effect" not appear.

\section{CONCLUSIONS AND SUGGESTIONS}

Based on big data of Baidu migration at the city level and cutting-edge econometric methods, we conduct a quantitative assessment of the impact of COVID-19 on economic activities. The main research conclusions are as follows:

The pandemic has a severe impact on economic activities in cities across the country. The estimation results of the difference-in-difference model (DID) demonstrate that the pandemic reduces the intensity of economic activity in cities nationwide by $48.4 \%$ in the two working days after the Spring Festival in 2020 compared with the same period in 2019. The results of the heterogeneity test suggest that for cities with large scale of population moving out and high dependence on migrant workers before the Spring Festival, the pandemic reduces the intensity of urban economic activity by $51.3 \%$ and $48.6 \%$ respectively in the two working days after the Spring Festival in 2020 compared with the same period in 2019. In other words, the negative effect of the pandemic on their economic activity is more prominent. On this basis, we adopt the event study method to reconfirm the finding that the pandemic has a negative impact on urban economic activities. Furthermore, the results of event study indicate that the economic activities of cities with large scale of population moving out and high dependence on migrant workers are affected by the pandemic to a greater extent.

Since the outbreak of COVID-19, domestic economic production activities and consumer markets have been severely impacted. In order to alleviate the adverse impact of the pandemic on economic operation, the central and local governments have successively introduced a series of policies and measures to ease the burden of enterprises and promote the gradual recovery of the economic operation in terms of increasing financial support, reducing and exempting relevant taxes and fees, etc. For cities of various types in different regions, factors such as the scale of population moving out before the Spring Festival and the dependence on migrant workers should be taken into account. Periodic resumption policies should be implemented according to the pandemic prevention and control status to establish an economic and social operation order corresponding to the pandemic prevention and control. The details are as follows:

The central and local governments have implemented the most stringent and comprehensive outbreak prevention and control measures, and have achieved remarkable results. However, reflecting on the facts of the outbreak and spread of the pandemic, it is clear that most cities across the country still have certain shortcomings in health care, disease prevention and control, and government emergency management. In addition to restricting population mobility and implementing strict preventive and control measures, it is more important to improve the emergency management system and economic shock adjustment programs, including public health events. In the process of building the emergency management system, it is crucial to ensure the openness and transparency of information, fully demonstrate the government's responsibility and decisiveness in responding to emergencies, enhance public confidence and avoid panic, and minimize the negative impact of the pandemic on economic performance.

In view of the weaknesses of the industrial chain revealed in the pandemic, timely remedies should be 
made to strengthen the management of the whole industrial chain, especially the upstream of the industrial chain. In addition, the government should guide the orderly and rational flow of labor and other factors in the industrial chain, while stabilizing the global industrial chain, strengthening international cooperation, improving the allocation efficiency of the industrial chain and enhancing the resilience of macroeconomic growth in the long run.

\section{AUTHORS' CONTRIBUTIONS}

Aili Du: Conceptualization, Methodology. Lei Wang: Data Curation, Formal analysis, WritingOriginal draft preparation. Aili Du: Project administration, Supervision, Validation. Aili Du: Writing-Reviewing and Editing.

\section{ACKNOWLEDGMENTS}

The authors gratefully acknowledge the financial support from the National Social Science Foundation of China (18FJY003). We also thank the anonymous referees for their helpful suggestions and corrections on the earlier draft of our paper, which improved the content.

\section{REFERENCES}

[1] Philipson T. Economic epidemiology and infectious diseases [R]. NBER Working Paper, 1999, No.7037.

[2] Gersovitz M, Hammer J S. The economical control of infectious diseases [J]. The Economic Journal, 2004, 114(492): 1-27.

[3] Zhu L. Start with the establishment of social medical insurance for infectious diseases[J]. Journal of Financial Research, 2003, (7): 16-24.

[4] Lei J. Legal system of the United States' compulsory medical treatment of infectious diseases and its enlightenment $[\mathrm{J}]$. Journal of Comparative Law, 2014, (6): 157-169.

[5] Krieger N. Epidemiology and the web of causation: has anyone seen the spider? [J]. Social Science and Medicine, 1994, 39(7): 887-903.
[6] Song L M. Public health practice in Chinese anthropology_-perspective of disciplinary cooperation based on anthropology and epidemiology $[\mathrm{J}]$. Journal of North Minzu University (Philosophy and Social Science), 2017, (5): 17-23

[7] Liu D H, Wang W G, Sun K. Scenario forecasting model and prevention-control measurements of important public health event based evolutionary game[J]. System Engineering - Theory \& Practice, 2012,32(5) :937-946.

[8] "SARS" and Public Management Research Group, Institute of Political Science, Chinese Academy of Social Sciences. Institutional analysis of SARS prevention and treatment in China[J]. Institute of Political Sciences, CASS, 2003, (3): 100-106.

[9] Mo Y C. One of the inspirations from the SARS crisis: the proper application of administrative guidance measures in public crisis management should be emphasized[J]. Theory and Reform, 2003, (5): 18-20.

[10] Wang L F, Ma J, Guo Z L. Public sector crisis management system: taking the SARS incident as an example $[\mathrm{J}]$. Chinese Public Administration, 2003, (7): 23-27.

[11] Zheng H S, Hong D Y. The "SARS" incident and China's social transition[J]. Population Research, 2003, (4): 47-50.

[12] Cai F, Wang M Y. The impact of "SARS" pandemic on employment in China[J]. Journal of Graduate School of Chinese Academy of Social Sciences, 2003, (4): 27-29.

[13] Wang J, Han K. Macro-control policy coordination: face up to the impact of "SARS" on the macroeconomy[J]. Chinese Public Administration, 2003, (7):12-16.

[14] Wang Q, Guo Z W, Han J L, et al. Analysis of the survey data of the cross-provincial inflow population from rural areas across the country during "SARS" [J]. Population Research, 2004, (2): 76-80. 